\title{
Dead regions in the cochlea at high frequencies: implications for the adaptation to hearing aids
}

\section{Angela Gordo ${ }^{1}$, Maria Cecília Martinelli Iório ${ }^{2}$}

Keywords: speech perception, self-assessment, hearing aids, sensorineural hearing loss.

\section{Summary}

\begin{abstract}
$I_{1}$ n patients with moderate to severe high-frequency hearing loss, cochlear damage may include "dead regions" where there are no functional inner hair cells and/or associated neurons. Aim: This study examines speech recognition in sensorineural impaired hearing patients with and without cochlear dead regions at high frequencies. Methods: a clinical and experimental study was made of thirty patients with sensorineural hearing loss that were classified into two groups: group 1 - included 15 subjects with hearing loss and no dead regions; and group 2 - included 15 subjects with dead regions in the cochlea at high frequencies. Patients undertook word recognition score and speech reception threshold tests, with and without background noise. The speech tests were done with and without hearing aids in two situations: program 1 - broadband amplification (bandwidth $8000 \mathrm{~Hz}$ ); and program 2 - amplification up to $2560 \mathrm{~Hz}$, without high frequency gain. Results: For subjects with no dead regions in the cochlea (group 1) performance improved with program 1. For subjects with dead regions in the cochlea (group 2) performance improved with program 2. Conclusions: Subjects with no dead regions in the cochlea benefited from high-frequency information. Subjects with dead regions in the cochlea benefited from reduced gain at high frequencies.
\end{abstract}

${ }^{1}$ Medical doctor, speech therapist, Doctor on The Science of Human Communication Disorders, trained at the Sao Paulo Federal University Paulista School of Medicine.

${ }^{2}$ Lecturer, Teacher in the discipline The Science of Human Communication Disorders at the Sao Paulo Federal University - Paulista School of Medicine. Head of the Discipline of Hearing Disorders at the Sao Paulo Federal University - Paulista School of Medicine. Sao Paulo Federal University - Paulista School of Medicine.

Address for correspondence: Angela Gordo - Av. Joao Ramalho, 505 ap.102 Vila Assuncao Santo Andre 09030-320.

Paper submitted to the ABORL-CCF SGP (Management Publications System) on April 13th, 2006 and accepted for publication on July 20 th, 2006. cod. 1840. 


\section{INTRODUCTION}

In audiology, sloping auditory sensorineural deficiency is the most common type/configuration of hearing loss associated with difficulty in understanding speech in noisy environments. Although hearing aids may increase the available acoustic information, not always a satisfactory improvement in speech recognition is attained. Some patients enjoy little or not benefit from amplification, particularly in cases of sloping hearing loss where a severe grade occurs at high frequencies.

An old concept is the relation between absence of benefit from hearing aids and functional reduction and complete loss of inner hair cells and/or neurons in certain regions of the cochlea. No clinical test, however, was done to identify the dead zones in the cochlea.

In these regions information generated by vibration of the basilar membrane is not transmitted to the central nervous system. If sufficiently intense, however, a tone with a corresponding frequency to that of the dead zone may be detected through apical or basal transmission of the vibration pattern by other functional regions of the cochlea. The vibration amplitude of the basilar membrane at a distant site will be lower than the dead zone amplitude. Broad band noise may thus mask that tone much more effectively than expected, as noise needs only to eliminate the response coming from the remote site. If the threshold needed to detect a tone in the presence of broad band noise is higher than that of the normal threshold, this alteration may indicate lack of inner hair cells and/or adjacent neurons, with a typical frequency that corresponds to the tone frequency, in other words, a dead zone. ${ }^{1}$

A few studies have related the difficulty that hearing loss imposes on speech recognition to the need for hearing speech at high sound pressure levels; these levels, however, may reduce the analytical capability of the normal cochlea. As hearing loss increases, certain frequencies do not support or even reduce the available information at other frequencies. Less amplification should therefore be prescribed for frequencies at with auditory thresholds are increased. ${ }^{2,5}$

Auditory resolution is the ability that inner ear structures and their associated neural systems have of generating patterns of neural activity that reflect spectral and time differences between sound information. The auditory nerve is organized, as is the basilar membrane: typically high frequency fibers originate from hair cells of the base of the cochlea, while low frequency fibers are at the apex of the cochlea. Fibers in the basal region respond synchronically to the presentation of a stimulus. Fibers in the apex are activated later (2 to $4 \mathrm{~ms}$ later).
Those that are activated simultaneously will contribute the most to the action potential of the whole nerve. Consequently this potential reflects mainly the response of high frequency fibers. ${ }^{3}$

Other authors 4 have proposed a clinical test to identify dead zones of the cochlea; this test is the threshold equalizing noise (TEN), which compares auditory thresholds investigated with and without ipsilateral masking. The TEN noise level has a spectrum that was elaborated to obtain equally masked thresholds at all frequencies, (125 Hz to $15000 \mathrm{~Hz}$ ), and is expressed as ERB (Equivalent Rectangular Bandwidth), which refers to the bandwidth of the auditory filter. Normal listeners have a small variation ( $2 \mathrm{~dB}$ to $3 \mathrm{~dB}$ ) between masked thresholds and noise levels. In patients with sensorineural hearing loss, dead zones of the cochlea are found when masked thresholds are at least $10 \mathrm{~dB}$ over absolute thresholds and $10 \mathrm{~dB}$ over the noise level. The results are confirmed by the measurement of psychophysical tuning curves. When the TEN test is positive, the peak of the tuning curve is displaced relative to the signal frequency. The authors emphasize that if dead zones are present, it may be useful to amplify a frequency range slightly above the dead zone; the reason being that amplification should aim at where hair cells can make use of it.

The effectiveness of hearing for intelligibility is affected by the sound pressure level of the signal (distortion level), the degree of hearing loss, the frequencies at which it takes place, and the sound information processing capability. ${ }^{5}$ As thresholds increase, the auditory efficiency decreases; this effect is amplified in high frequency hearing loss. The practical implication of this concept for hearing aid adaptation is that increased amplification should be applied where thresholds are less affected.

Based on these thoughts, the aim of this study was to verify the benefit of high frequency amplification for speech recognition in patients with sloping sensorineural hearing loss with or with no dead zones in the cochlea.

\section{METHODS}

The procedures used in this study were described to the Research Ethics Committee and approved under code number 0235/04. Participants signed a free informed consent form containing the necessary information before undergoing the tests. We assessed 30 subjects (14 women and 16 men) with sloping, bilateral, and symmetrical sensorineural hearing loss. Identification of dead zones of the cochlea was done using the TEN(NA) test, 6 2004 version. Two groups were defined based on these results: group 1 - 15 subjects with no dead zones of the 
Table 1. Distribution of the sample population as to gender, age, complaint, duration of complaint, and use of hearing aids in group 1.

\begin{tabular}{|c|c|c|c|c|}
\hline Sex & Age & Complaint & Duration & Hearing aids \\
\hline M & 45 & Sudden hearing loss & 40 years & 8 months \\
\hline $\mathrm{F}$ & 49 & Listens but does not understand speech & 3 years & Adapting \\
\hline M & 49 & Progressive hearing loss / tinnitus & 6 years & 1 year \\
\hline M & 58 & Progressive hearing loss / tinnitus & 3 years & Adapting \\
\hline M & 66 & Progressive hearing loss / tinnitus & 10 years & 1 year \\
\hline M & 68 & Progressive hearing loss & 6 years & 6 years \\
\hline M & 69 & Progressive hearing loss & 2 years & 1 month \\
\hline M & 71 & Progressive hearing loss / tinnitus & 6 years & Adapting \\
\hline $\mathrm{F}$ & 72 & Progressive hearing loss & 5 years & Adapting \\
\hline M & 73 & Progressive hearing loss & 3 years & Adapting \\
\hline M & 74 & Does not understand speech & 20 years & 3 months \\
\hline $\mathrm{F}$ & 75 & Progressive hearing loss & 10 years & 3 months \\
\hline M & 75 & Progressive hearing loss & 22 years & 4 months \\
\hline $\mathrm{F}$ & 76 & Progressive hearing loss & 2 years & 1 month \\
\hline M & 83 & Does not understand speech / tinnitus & 20 years & 1 year \\
\hline
\end{tabular}

Table 2. Distribution of the sample population as to gender, age, complaint, duration of complaint, and use of hearing aids in group 2.

\begin{tabular}{|c|c|c|c|c|}
\hline Sex & Age & Complaint & Duration & Hearing aids \\
\hline $\mathrm{F}$ & 19 & Would like to hear better & 16 years & 1 year \\
\hline $\mathrm{F}$ & 24 & Does not understand speech / tinnitus & 12 years & Adapting \\
\hline $\mathrm{F}$ & 26 & Does not understand speech & 6 years & Adapting \\
\hline $\mathrm{F}$ & 34 & Does not understand speech & 10 years & Adapting \\
\hline M & 35 & Does not hear/discomfort for loud sounds & 10years & Adapting \\
\hline $\mathrm{F}$ & 36 & Does not understand speech & 12 years & 9 months \\
\hline $\mathrm{F}$ & 43 & Progressive hearing loss / tinnitus & 4 years & 1 mês \\
\hline M & 50 & Constant tinnitus & 3 years & 4 months \\
\hline M & 54 & Does not hear well / tinnitus & 5 years & Adapting \\
\hline M & 57 & Does not hear well / tinnitus & 10 years & Adapting \\
\hline M & 64 & Progressive hearing loss / tinnitus & 20 years & Adapting \\
\hline $\mathrm{F}$ & 69 & Progressive hearing loss / tinnitus & 8 years & 2 months \\
\hline $\mathrm{F}$ & 73 & Does not understand speech / tinnitus & 15 years & Adapting \\
\hline $\mathrm{F}$ & 75 & Does not understand speech / tinnitus & 30 years & 1 month \\
\hline $\mathrm{F}$ & 75 & Does not hear well / does not understand speech & 20 years & 2 years \\
\hline
\end{tabular}

cochlea, and group 2 - 15 subjects with dead zones of the cochlea at high frequencies.

Tables 1 and 2 show the distribution of the sample population including gender, age, complaint, duration of the complaint, and use of hearing aids (if a user for how long or if in the selection/adaptation process) for group 1 and 2 .

The TEN test was applied using a two channel audiometer to control separately the stimulus (pure tone) and noise. The audiometer was coupled to a CD player. Auditory thresholds were measured in $2 \mathrm{~dB}$ intervals at 500, 750, 1000, 1500, 2000, 3000, and $4000 \mathrm{~Hz}$ in each ear separately, using TDH49 earphones, initially without masking, followed by an ipsilateral TEN noise at $70 \mathrm{~dB}$ $\mathrm{NA} / \mathrm{ERB}$. If this level was not sufficient to mask the absolute threshold, we would gradually increase the intensity up to $85 \mathrm{~dB}$ NA/ERB (maximum noise level tolerated by our patients). When the masked threshold was $10 \mathrm{~dB}$ or 
more over the absolute threshold and the noise level, we would give the result as suggesting dead zones for the tested frequency.

We then proceeded with speech recognition tests in quite and with background noise, presenting the stimuli in an acoustic room, always using the same loudspeaker that the patient sat facing at a distance of 1 meter and azimuth $0^{\circ}$.

We used a digital Siemens Signia HdO+ behindthe-ear hearing aid with eight independently programmable channels for different frequency ranges to observe performance according to the amplified signal. Adaptation of hearing aids was binaural in all subjects. There were two programs: program 1 was amplification at a wide range of low frequencies (between $100 \mathrm{~Hz}$ and $8000 \mathrm{~Hz}$ ), and program 2 had no gain at high frequencies (over 2560 $\mathrm{Hz}$ ). The cut-off frequency for program 2 was approximately $2000 \mathrm{~Hz}$, which was selected based on research that reports benefits from amplification of 1.7 times the dead zone limit frequency (roughly one octave higher). ${ }^{7}$ Although this measurement was not done precisely in our study, results were positive for dead zones starting between 1000 and $1500 \mathrm{~Hz}$ in most cases.

The speech material used for the Percentage Index of Speech Recognition (PISR) survey was a list of 25 phonetically balanced monosyllables ${ }^{8}$ recorded in four difference sequences. The PISR was assessed in quite and with background speech noise. With no hearing aids, speech intensity was set as the most comfortable level reported by patients. With hearing aids, speech and noise were set at $65 \mathrm{~dB} \mathrm{~A}$. We then investigated the Sentence Recognition Threshold in quiet (SRTQ) and in the presence of background noise (SRTN) using five lists containing 10 phonetically balanced sentences.9 For the SRTQ test we presented the first sentence using the best ear speech reception threshold (obtained by earphones). Noise was set at $65 \mathrm{~dB}$ A for the SRTN test, and the first sentence was presented always in a zero signal-to-noise ratio. Both tests (PISR and SRTQ/N) were investigated under three different conditions: unaided, aided using program 1 , and aided using program 2 ; the sequence of procedures and the selected lists were alternated at each presentation.

We then applied the Abbreviated Profile of Hearing Aid Benefit $(\mathrm{APHAB})^{10}$ questionnaire to assess communication difficulties in daily situations. This questionnaire includes 24 items on three communication subscales related to the acoustic environment and one subscale on discomfort for intense sounds, namely: ease of communication, reverberation, background noise and aversiveness of sounds. As not all subjects used hearing aids, we used only the responses for unaided performance. Participants chose the option for each item that came closest to their everyday experience: A-always (99\%); B-almost always (87\%); C-generally (75\%); D-half-the-time (50\%); E-occasionally (25\%); F-seldom (12\%); G-never (1\%). Results were quantified to reach a score for each subscale.

We used non-parametric comparison tests to analyze our results statistically. Since the sample was relatively small, our significance level was set at 0.07 (7\%).

Masked thresholds were never over $10 \mathrm{~dB}$ above the noise level (from 70 to $85 \mathrm{~dB} \mathrm{NA} / \mathrm{ERB}$ ) in all patients; the maximum difference between them was $6 \mathrm{~dB}$ NA. Generally only one noise level (70 dB NA/ERB) was enough for the TEN(NA) test. When the audiometric threshold was above $60 \mathrm{~dB}$ at a specific frequency, we used $10 \mathrm{~dB}$ over this threshold to define the minimum masking level.

When masked auditory thresholds obtained by TEN(NA) testing of group 2 exceeded one or more of the absolute thresholds and the noise level by $10 \mathrm{~dB}$, we considered the result as positive for dead zones of the cochlea at high frequencies. We used two or three noise levels due to the degree of hearing loss at these frequencies. Various patients had a $10 \mathrm{~dB}$ or more change compared to the absolute threshold when the noise level was below this threshold, where masking would theoretically be insufficient to change the threshold.

In subjects with no dead zones of the cochlea we found that the PISR in quiet and in the presence of background noise improved significantly when using program 1 compared to the unaided and aided conditions of program 2.

When dead zones of the cochlea were present we found that the PISR in quiet and in the presence of background noise improved significantly when using programs 1 and 2 in unaided conditions, and that program 2 showed significantly improved results compared to program 1.

SRTQ was significantly improved in group 1 when using program 1 in unaided condition and to program 2. In the presence of background noise (SRTN) we can say that there was a trend towards a difference between both programs, as the statistical analysis revealed that the p-value was close to the acceptable limit. There were no significant differences between program 2 unaided and aided results.

SRTQ and SRTN were significantly improved in group 2 when using program 2 and unaided conditions and when using program 1 and aided conditions.

There was a significant difference between groups only in environments that favored communication; in this condition the most significant difficulty was found in group 2. 
Table 3. Auditory thresholds (dB NA) and mean differences between masked thresholds and the noise level in group 1.

\begin{tabular}{|c|c|c|c|c|c|c|c|c|}
\hline \multicolumn{9}{|c|}{ Frequencies (kHz) } \\
\hline Subjects & 0.5 & 0.75 & 1 & 1.5 & 2 & 3 & 4 & \\
\hline 1 & $\mathrm{RE}$ & 32 & 28 & 34 & 36 & 36 & 56 & 60 \\
\hline \multirow[t]{2}{*}{2} & RE & 24 & 26 & 32 & 40 & 38 & 46 & 60 \\
\hline & LE & 52 & 52 & 50 & 60 & 56 & 58 & 66 \\
\hline 3 & LE & 20 & 20 & 20 & 38 & 56 & 62 & 52 \\
\hline \multirow[t]{2}{*}{4} & RE & 16 & 20 & 20 & 26 & 36 & 50 & 60 \\
\hline & LE & 24 & 24 & 26 & 50 & 46 & 52 & 54 \\
\hline \multirow[t]{2}{*}{5} & $\mathrm{RE}$ & 18 & 26 & 30 & 46 & 48 & 54 & 60 \\
\hline & LE & 26 & 26 & 32 & 44 & 48 & 50 & 58 \\
\hline 7 & LE & 18 & 24 & 36 & 36 & 54 & 54 & 60 \\
\hline \multirow[t]{2}{*}{8} & $\mathrm{RE}$ & 16 & 20 & 24 & 56 & 60 & 58 & 70 \\
\hline & LE & 16 & 20 & 24 & 50 & 54 & 56 & 64 \\
\hline \multirow[t]{2}{*}{9} & $\mathrm{RE}$ & 24 & 34 & 50 & 50 & 50 & 54 & 70 \\
\hline & LE & 28 & 32 & 42 & 42 & 42 & 48 & 60 \\
\hline \multirow[t]{2}{*}{10} & $\mathrm{RE}$ & 24 & 22 & 32 & 38 & 44 & 58 & 60 \\
\hline & LE & 14 & 12 & 18 & 34 & 34 & 60 & 54 \\
\hline 11 & $\mathrm{RE}$ & 32 & 38 & 38 & 50 & 50 & 58 & 66 \\
\hline 14 & LE & 30 & 30 & 32 & 32 & 38 & 42 & 52 \\
\hline \multirow[t]{2}{*}{15} & $\mathrm{RE}$ & 18 & 14 & 8 & 42 & 54 & 54 & 60 \\
\hline & LE & 38 & 28 & 52 & 54 & 52 & 64 & 64 \\
\hline \multirow[t]{2}{*}{ Mean } & $\mathrm{RE}$ & 4.3 & 4.3 & 4.9 & 5.7 & 5.7 & 5.3 & 3.8 \\
\hline & LE & 2.0 & 3.2 & 4.1 & 5.2 & 5.1 & 5.0 & 4.5 \\
\hline
\end{tabular}

Key: RE - right ear; LE - left ear 
Table 4. Auditory thresholds (dB NA) and mean differences between masked thresholds and the noise level in group 2.

\begin{tabular}{|c|c|c|c|c|c|c|c|c|}
\hline \multicolumn{9}{|c|}{ Frequencies (kHz) } \\
\hline Subjects & 0.5 & 0.75 & 1 & 1.5 & 2 & 3 & 4 & \\
\hline \multirow[t]{2}{*}{1} & $\mathrm{RE}$ & 6 & 54 & 64 & $64^{*}$ & $66^{*}$ & AR & $A R$ \\
\hline & LE & 8 & 52 & 64 & $66^{*}$ & $86^{*}$ & AR & AR \\
\hline \multirow[t]{2}{*}{2} & $\mathrm{RE}$ & 6 & 28 & 52 & $64^{*}$ & $66^{*}$ & $64^{*}$ & $66^{*}$ \\
\hline & LE & 8 & 14 & 46 & $70^{*}$ & $68^{*}$ & $64^{*}$ & $64^{*}$ \\
\hline \multirow[t]{2}{*}{3} & $\mathrm{RE}$ & 22 & 18 & $82^{*}$ & $82^{*}$ & $90^{*}$ & AR & AR \\
\hline & LE & 20 & 26 & $74^{*}$ & $82^{*}$ & $80^{*}$ & $84^{*}$ & $82^{*}$ \\
\hline \multirow[t]{2}{*}{4} & $\mathrm{RE}$ & 42 & 52 & 60 & $86^{*}$ & $82^{*}$ & $80^{*}$ & $86^{\star}$ \\
\hline & LE & 34 & 52 & 54 & $80^{*}$ & $82^{*}$ & $76^{*}$ & $82^{*}$ \\
\hline \multirow[t]{2}{*}{5} & $\mathrm{RE}$ & 44 & 72 & $78^{*}$ & $78^{*}$ & $78^{*}$ & $94^{*}$ & AR \\
\hline & LE & 38 & 48 & $74^{*}$ & $\mathrm{AR}$ & AR & $96^{*}$ & AR \\
\hline \multirow[t]{2}{*}{6} & $\mathrm{RE}$ & 88 & 86 & $98^{*}$ & $94^{*}$ & $94^{*}$ & $90^{*}$ & $80 *$ \\
\hline & LE & 32 & 42 & $72^{\star}$ & $92^{*}$ & $92^{*}$ & AR & AR \\
\hline \multirow[t]{2}{*}{7} & $\mathrm{RE}$ & 42 & 50 & 62 & 82 & $92^{*}$ & AR & AR \\
\hline & LE & 60 & 62 & 68 & $80^{*}$ & $86^{*}$ & $82^{*}$ & AR \\
\hline \multirow[t]{2}{*}{8} & $\mathrm{RE}$ & 4 & 8 & 14 & 36 & $62^{*}$ & $58^{*}$ & $58^{*}$ \\
\hline & LE & 4 & 12 & 16 & $58^{*}$ & $60^{*}$ & $58^{*}$ & $56^{\star}$ \\
\hline \multirow[t]{2}{*}{9} & $\mathrm{RE}$ & 46 & 56 & 74 & $88^{*}$ & AR & AR & AR \\
\hline & LE & 60 & 68 & $88^{*}$ & $\mathrm{AR}$ & $A R$ & AR & AR \\
\hline \multirow[t]{2}{*}{10} & $\mathrm{RE}$ & 50 & 68 & $86^{*}$ & $\mathrm{AR}$ & AR & $A R$ & AR \\
\hline & LE & 46 & 62 & 68 & 74 & $80^{*}$ & 80 & $90 *$ \\
\hline \multirow[t]{2}{*}{11} & $\mathrm{RE}$ & 26 & 20 & 18 & 44 & $58^{*}$ & $98^{*}$ & $90 *$ \\
\hline & LE & 10 & 8 & 10 & 32 & 56 & $82^{*}$ & $A R$ \\
\hline \multirow[t]{2}{*}{12} & $\mathrm{RE}$ & 46 & 64 & $70^{*}$ & $70^{*}$ & $84^{*}$ & $74^{\star}$ & $92^{*}$ \\
\hline & LE & 46 & 70 & $74^{*}$ & $84^{*}$ & $82^{*}$ & $A R$ & AR \\
\hline \multirow[t]{2}{*}{13} & $\mathrm{RE}$ & 42 & 66 & 70 & $80^{*}$ & $A R$ & AR & AR \\
\hline & LE & 50 & 62 & $72^{\star}$ & $96^{*}$ & AR & AR & $A R$ \\
\hline \multirow[t]{2}{*}{14} & $\mathrm{RE}$ & 18 & 28 & 30 & $40^{*}$ & $52^{*}$ & $64^{*}$ & $68^{*}$ \\
\hline & LE & 20 & 18 & 28 & $30^{*}$ & $38^{*}$ & $62^{*}$ & $64^{*}$ \\
\hline \multirow[t]{2}{*}{15} & $\mathrm{RE}$ & 54 & 62 & 68 & $78^{*}$ & $84^{*}$ & $96^{*}$ & $94^{*}$ \\
\hline & LE & 48 & 56 & 66 & $72^{*}$ & $86^{*}$ & $86^{\star}$ & $84^{\star}$ \\
\hline \multirow[t]{2}{*}{ Mean } & $\mathrm{RE}$ & 3.9 & 2.6 & 6.4 & 13.2 & 15.1 & 18.5 & 19.5 \\
\hline & LE & 3.9 & 4.6 & 8.5 & 13.2 & 14.8 & 17.2 & 19.5 \\
\hline
\end{tabular}

Key: RE - right ear; LE - left ear; AR - absence of response at the maximum pure tone intensity (102 dB NA).

* presence of dead zone of the cochlea at the frequency tested. 


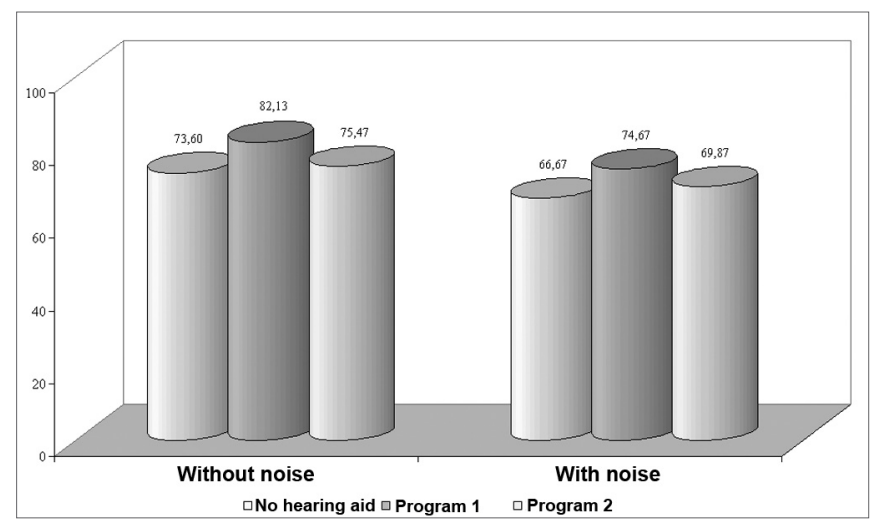

Figure 1. Chart showing the percentage index of speech recognition in quiet and with background noise in group 1.

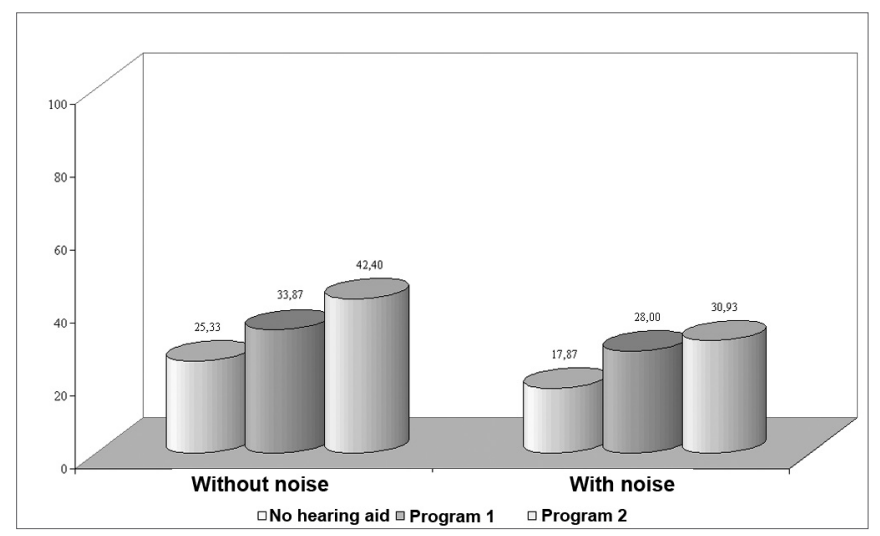

Figure 2. Chart showing the percentage index of speech recognition in quiet and with background noise in group 2 .

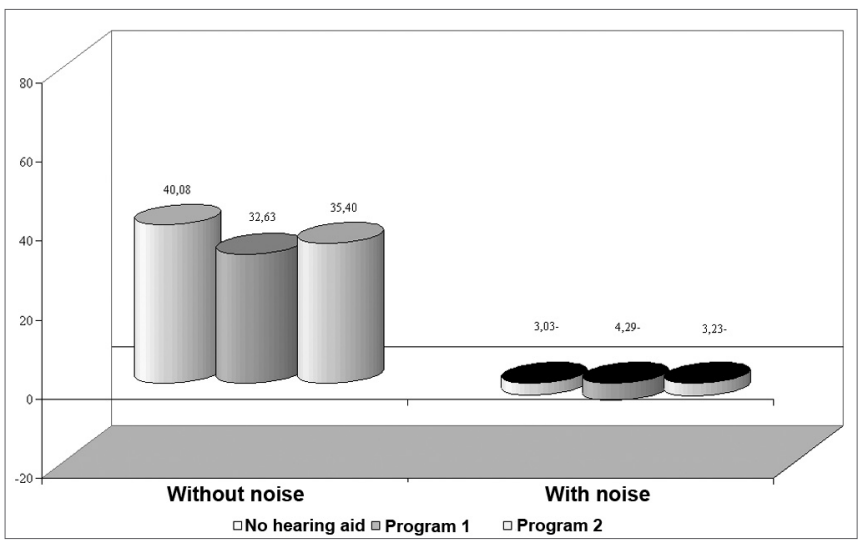

Figure 3. Chart showing the sentence recognition threshold in quiet (SRTQ) and with background noise (SRTN) in group 1.

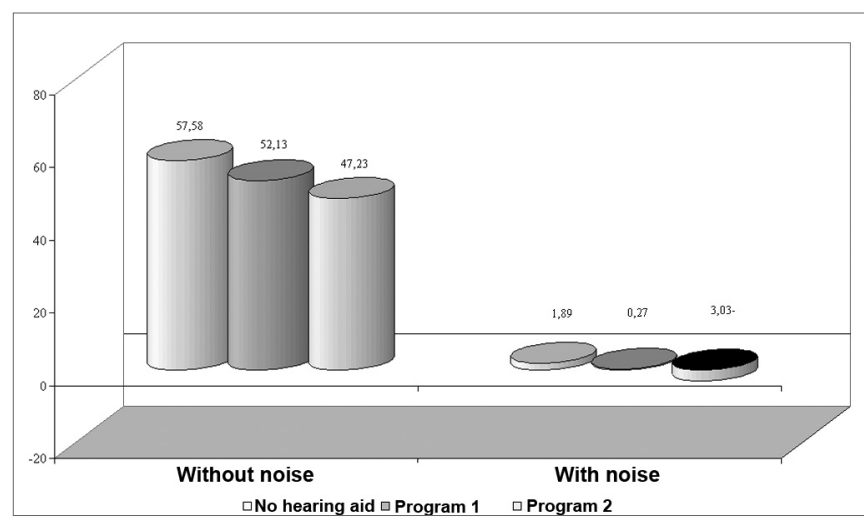

Figure 4. Chart showing the sentence recognition threshold in quiet (SRTQ) and with background noise (SRTN) in group 2.

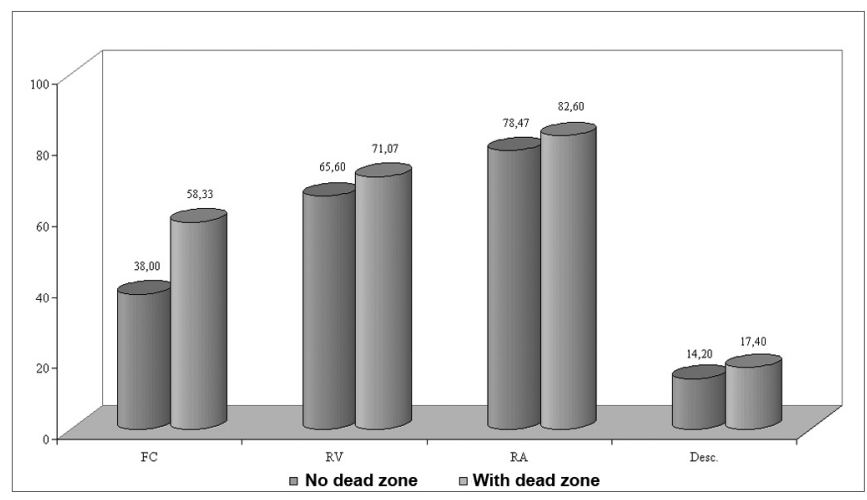

Figure 5. Chart showing the communication difficulty (\%) in various sound environments and aversiveness to sounds according to the APHAB questionnaire in groups 1 and 2 .

\section{DISCUSSION}

The degree of high frequency hearing loss and the percentage index of speech recognition already suggested significant differences between groups 1 and 2 before we identified dead zones of the cochlea. We may say that a negative result for dead zones of the cochlea corresponded to the expected pattern in group 1 , due to the audiometric sloping configuration but with no threshold differences over $50 \mathrm{~dB}$ obtained in successive octaves of tested frequencies, and the absence of thresholds over $90 \mathrm{~dB}$ NA at high frequencies (Table 3) ${ }^{11}$ We found a positive result for dead zones at high frequencies over $1500 \mathrm{~Hz}$ in group 2 in most cases (Table 4). Many patients reported hearing a different sound, similar to a hiss, when the pure tone frequency was associated with the dead zone. ${ }^{12}$

The percentage index of speech recognition in group 1 showed a significant improvement when using hearing aids with program 1 (sound amplification in a wide frequency range from $100 \mathrm{~Hz}$ to $8000 \mathrm{~Hz}$ ) compared to program 2 (restricted amplification from $100 \mathrm{~Hz}$ to 2560 $\mathrm{Hz}$ ), both in quiet and in the presence of background noise (Figure 1). Thus, if there are no dead zones of the 
cochlea, high frequency information effectively contributes to speech intelligibility. $5,7,13,14$

The percentage index of speech recognition in group 2 was significantly improved by using hearing aids with programs 1 and 2 compared to the unaided condition, both in quiet and in the presence of background noise (Figure 2), although the highest benefit was seen with program 2. Sound amplification in a restricted frequency range - with a lower gain at frequencies in which hearing loss is most severe - favored information use where audibility is useful. 2,5,14,15

We may assume that patients with dead zones of the cochlea at high frequencies are used to a perception of filtered speech, as their hearing would operate as a low-pass filter. This could explain the benefit difference between each program for groups 1 and 2. Subjects with no dead zones, that effectively use high frequency information, are more affected by removal of these high frequencies. ${ }^{14}$

Comparing both programs, we observed that group 2 subjects reported increased clarity of sound and absence of hissing with program 2 . We believe that the presence of dead zones at high frequencies reduce sound distortion by not amplifying those frequencies. Vibration generated in a dead zone is detected by another region; little useful information, therefore, is transmitted from the affected site. Furthermore, when the typical frequency of a region is different from that of the stimulus, detection of intense sound corresponding to these regions is altered. ${ }^{6}$

Group 1 subjects performed better in the sentence recognition threshold test in quiet and in the presence of background noise when using program 1 compared to program 2 (Figure 3 ). There was no significant performance difference in program 2 with or without hearing aids in the presence of background noise; in this condition, program 2 offered practically no benefit. Once again, these results may be associated with the use of high frequency information to attain speech intelligibility.

Sentence recognition thresholds in quiet and in the presence of background noise (Figure 4) for group 2 benefited from both programs, where benefits from program 2 were more significant than those from program 1. The etiology and the time during which adequate auditory stimuli at high frequencies were absent should be taken into account when using high frequency amplification for conditions of marked hearing loss at these frequencies. ${ }^{5}$ Ten subjects with dead zones (66.7\% of group 2) had a history of hearing loss for ten years or more, a relatively long period of absent auditory stimuli, which may have contributed to improved results with amplification of a reduced frequency range.

The APHAB questionnaire (Figure 5), used to compare both groups, revealed that there was a significant difference between groups only in the ease of communi- cation environment, where group 2 had more communication difficulties. This result confirms the less favorable performance of group 2 in speech tests. This performance is related to the severity of hearing loss and the presence of dead zones of the cochlea.

Over half of group 2 subjects were going through hearing aid selection procedures just by taking part of this study, as previous adaptation attempts had been unsuccessful. In another paper the superior percentage index of speech recognition in patients with no dead zones of the cochlea was related to a higher acceptance rate of hearing aids (94.1\%). In the presence of dead zones the acceptance rate was $21.4 \%$. During the tests patients in group 2 reported improved sound quality with program 2 , and that they wished to attempt adaptation once again.

Observing the region in which auditory thresholds were preserved most in group 2, we could question whether amplification of sounds corresponding to this area could truly benefit these patients. We know that there is also loss of low frequency phase/synchronization components together with loss of high frequency information. ${ }^{3,17}$ Thus, gain at these frequencies is very important. We believe that the strategy of limiting sound amplification in affected areas where amplification would offer little benefit is the most adequate choice for the auditory rehabilitation of patients with dead zones of the cochlea.

\section{CONCLUSION}

Based on a critical analysis of our results, we reached the following conclusions:

1. In the absence of dead zones of the cochlea, improved speech recognition performance is reached with amplification over a wide frequency range.

2. In the presence of dead zones of the cochlea at high frequencies, sound amplification of a restricted frequency range, avoiding gain at high frequencies, leads to the best speech recognition performance.

\section{REFERENCES}

1. Moore BCJ, Glasberg BR. A model of loudness perception applied to cochlear hearing loss (1997) apud Moore, BCJ, Glasberg, BR. Br J Audiol 1998;32:317-35.

2. Hogan CA, Turner CW. High-frequency audibility:benefits for hearingimpaired listeners. J Acoust Soc Am 1998;104(1):432-41.

3. Bess FH, Humes LE. Estrutura e função do sistema auditivo. In:Bess FH, Humes LE. Fundamentos de Audiologia. Trad. de Marcos AG Domingues. Porto Alegre: Artmed; 1998. p.64-105.

4. Moore BCJ, Huss M, Vickers DA, Glasberg BR, Alcantara JI. A test for the diagnosis of dead regions in the cochlea. Br J Audiol 2000;34:20524

5. Ching TYC, Dillon H, Katsch R, Byrne D. Maximizing effective audibility in hearing aid fitting. Ear \& Hear 2001;22(3):212-24.

6. Moore BCJ. Dead regions in the cochlea: conceptual foundations, diagnosis, and critical applications. Ear \& Hear 2004;2(2):98-116.

7. Vickers DA, Moore BCJ, Baer T. Effects of low-pass filtering on the intelligibility of speech in quiet for people with and without dead 
regions at high frequencies. J Acoust Soc Am 2001;110(2):1164-75.

8. Pen MG, Mangabeira-Albernaz PL. Lista de monossílabos para discriminação vocal. In: Mangabeira-Albernaz PL, Ganança MM ed. Surdez neurossensorial. São Paulo: Editora Moderna; 1976.p.20.

9. Costa MJ. Desenvolvimento de listas de sentenças em português [tese]. São Paulo:Universidade Federal de São Paulo;1997.

10. Cox RM, Alexander GC. The abbreviated profile of hearing aid benefit. Ear \& Hear 1995; 16:176-86.

11. Moore BCJ. Dead regions in the cochlea: diagnosis, perceptual consequences and implications for the fitting of hearing aids. Trends in amplif 2001;5:1-34.

12. Moore BCJ, Vickers DA, Plack CJ, Oxenham AJ. Inter-relationship between different psychoacoustic measures assumed to be related to the cochlear active mecanism. J Acoust Soc Am 1999;106(5):276178.
13. Baer T, Moore BCJ, Kulk K. Effects of low pass filtering on the intelligibility of speech in noise for people with and without dead regions at high frequencies. J Acoust Soc Am 2002;112(3):1133-44.

14. Vestergaard M. Dead regions in the cochlea:implications for speech recognition and applicability of articulation index theory. Internat J Audiol 2003;42 (5):249-61.

15. Ching TYC, Dillon H, Byrne D. Speech recognition of hearing-impaired listeners:predictions from audibility and the limited role of high-frequency amplification. J Acoust Soc Am 1998;103:1128-40.

16. Eguti EY. Identificação das zonas mortas na cóclea utilizando a técnica de mascaramento com ruído branco [tese]. São Paulo:Universidade Federal de São Paulo;2002.

17. Horwitz AR, Dubno JR, Ahlstrom JB. Recognition of low-pass-filtered consonants in noise with normal and impaired high-frequency hearing. J Acoust Soc Am 2002;111 (1):409-16. 